\title{
Machine Translation and its philosophical accounts
}

\author{
Stelios Piperidis \\ Institute for Language and Speech Processing, \\ "Athena" Research Centre \\ spipeilsp.gr
}

\begin{abstract}
This paper attempts to explore the interrelation between philosophical accounts of language and respective technological developments in the field of human language technologies. In doing so, it focuses on the interaction between analytical philosophy and machine translation development, trying to draw the emerging methodological analogies.
\end{abstract}

\section{Introduction}

Philosophical accounts of science and respective technological development bear a tight interrelation and continuous interplay. Likewise, philosophical investigations of language bear their own implications on how technology processing language, in monolingual or multilingual settings, evolves.

In the multilingual setting, machine translation feasibility, its presuppositions and implications, brings forth a range of questions, applicable to human translation as well, including, but not limited to, linguistic and ontological relativity, indeterminacy of translation, inscrutability of reference, representational function of language, the problem of meaning.

Bar Hillel's claims on the infeasibility of machine translation and the Sapir-Whorf hypothesis with the linguistic determinism and relativity principles, partly backed up by Quine's ontological relativity and later Wittgenstein's private language and variability of language games have haunted the way of thinking in machine translation development. Indeterminacy, relativity, and the consequent abolition of the one gold translation idea, however, as well as the necessity for frameworks integrating pragmatic and behavioural data in translation have played their role in advancing machine translation design paving the way for observed paradigm shifts at all stages of development.

In broadly dividing machine translation history in the rule-based and corpus-based eras, in the 50 's and 80's respectively, one can draw the analogies that would rather point to a tight interaction between philosophical accounts and machine translation paradigms.

Early contemporary analytic philosophy, through conceptual, reference-bound analysis and compositionality principles, provided the foundation for representational, rule and knowledge-based approaches of early machine translation, from 50 's through the 80 's. The turn, in analytic philosophy, to an understanding of meaning though use, to pragmatics and behaviourism, may be paired and seen as laying the foundation for the machine translation paradigm shift observed in the 80's. In this pairing, it is the use of the much required parallel (or comparable) translation data that could be seen as constituting the behavioural data base, with each alignment function being conceived of as the result of a radical translation process, where a source language sentence provides the sensory data and a target language sentence provides the linguistic observation. In such a framework, this aligned data source does provide the "translation manual", which after a series of inductive operations does converge to a potentially usable set of translation relations.

Along this line, we will discuss, in this talk, the continuous relations between analytic philosophy, linguistic science and human language technologies. Such relations, direct or indirect, can be bi-directional and can possibly work towards better understanding and facilitating the virtuous cycle between language technology and its theoretical underpinnings. 